\title{
ANALYTIC PROPERTIES OF A CERTAIN MULTIPLE DIRICHLET SERIES
}

\author{
Takuya OKAMOTO
}

(Received 12 January 2011)

Abstract. We consider a certain multiple Dirichlet series that is a generalization of that introduced by Masri 2005, and we prove the meromorphic continuation to the whole space. Also, using certain functional relations and the technique of changing variables, we prove that 'the possible singularities' are indeed 'the true singularities'.

\section{Statement of results}

Let $r$ be a fixed positive integer and $s=\sigma+i t$ be a complex variable, and define a Dirichlet series with complex coefficients $a(n)$ by

$$
L(s)=\sum_{n=1}^{\infty} a(n) n^{-s} .
$$

In the present paper we suppose that $L(s)$ satisfies the following two conditions:

(i) there exists a number $\mu>0$, such that $a(n) \ll n^{\mu}$, hence $L(s)$ is convergent absolutely for $\sigma>\mu+1$;

(ii) $L(s)$ has a meromorphic continuation to $\mathbb{C}$ with a pole at $s=1$ of order one.

In the present paper we introduce the multiple Dirichlet series with complex coefficients

$$
L_{r, i}\left(\left(s_{1}, \ldots, s_{r}\right) ; a\right)=\sum_{1 \leq m_{1}<\cdots<m_{r}<\infty} \cdots \sum_{i} a\left(m_{i}\right) m_{1}^{-s_{1}} m_{2}^{-s_{2}} \cdots m_{r}^{-s_{r}},
$$

where $s_{k}=\sigma_{k}+i t_{k}(1 \leq k \leq r)$ are complex variables.

Let $F_{k}(1 \leq k \leq r)$ be number fields, $\mathcal{O}_{F_{k}}$ be their rings of integers, and for an integral ideal $I_{k}$ in $F_{k}$, put $N_{F_{k} / \mathbb{Q}} I_{k}=\left[\mathcal{O}_{F_{k}}: I_{k}\right]$. Then, Masri [2] introduced the multiple Dedekind zeta functions

$$
\sum_{\substack{1 \leq N_{F_{1} / \mathbb{Q}} I_{1}<\cdots<N_{F_{r} / \mathbb{Q}} I_{r}<\infty \\ I_{1} \subset \mathcal{O}_{F_{1}}, I_{2} \subset \mathcal{O}_{F_{2}}, \ldots, I_{r} \subset \mathcal{O}_{F_{r}}}} N_{F_{1} / \mathbb{Q}} I_{1}^{-s_{1}} N_{F_{2} / \mathbb{Q}} I_{2}^{-s_{2}} \cdots N_{F_{r} / \mathbb{Q}} I_{r}^{-s_{r}} .
$$

Wojtkowiak [7] and Zhao [10] studied a similar function to (1.2). When $F_{k}=\mathbb{Q}(1 \leq k \leq r)$, the multiple Dedekind zeta function (1.2) is nothing but the well-known Euler-Zagier sum [8]

$$
\zeta_{E Z, r}\left(s_{1}, \ldots, s_{r}\right)=\sum_{1 \leq m_{1}<\cdots<m_{r}<\infty} \cdots m_{1}^{-s_{1}} m_{2}^{-s_{2}} \cdots m_{r}^{-s_{r}} .
$$

Also, the following analytic properties of (1.3) are known.

2000 Mathematics Subject Classification: Primary 11M41; Secondary 32D15.

Keywords: multiple Dirichlet series; analytic continuation; Mellin-Barnes formula; true singularities.

(c) 2012 Faculty of Mathematics, Kyushu University 
THEOREM 1.1. ([1, Theorem 1], [4, Theorem 1])

(1) The series (1.3) can be continued meromorphically, as a function in $s_{1}, \ldots, s_{r}$, to the whole $\mathbb{C}^{r}$-space.

(2) The function (1.3) is holomorphic except for the singularities located only on

$$
\begin{aligned}
s_{r} & =1, \\
s_{r}+s_{r-1} & =2,1,-2 n \quad\left(n \in \mathbb{N}_{0}\right), \\
s_{r}+s_{r-1}+s_{r-2} & =3-n \quad\left(n \in \mathbb{N}_{0}\right), \\
\cdots & \\
s_{r}+\cdots+s_{1} & =r-n \quad\left(n \in \mathbb{N}_{0}\right) .
\end{aligned}
$$

We fix $i(1 \leq i \leq r)$ in (1.1). The multiple Dirichlet series (1.1) can be seen as a special case of (1.2) by putting $F_{k}=\mathbb{Q}(k \neq i)$ and

$$
a\left(m_{i}\right)=\left|\left\{\{0\} \neq I \subset \mathcal{O}_{F_{i}} \mid N_{F_{i} / \mathbb{Q}} I=m_{i}\right\}\right| .
$$

Hence (1.1) is a generalization of the Euler-Zagier sum of the form (1.3). In the case $i=1, r$, Masri [2] proved the meromorphic continuation of them to the whole $\mathbb{C}^{r}$-space and gave linear subvarieties comprising their singularities. There are various methods for the proof of analytic continuation of (1.3) [1, 3, 9]. Masri [2] used the method of Akiyama et al [1].

The purpose of the present paper is to discuss analytic properties of (1.1). Our proof of their analytic continuation in the present paper is based on the method of Matsumoto [3], which uses the Mellin-Barnes integral. However, in our case, a simple analogue of Matsumoto's argument is not sufficient. A new idea in the present paper is to transform the sum suitably to get a form for which the Mellin-Barnes integral method of separating variables can be applied successfully (see (2.3) and (2.4)).

The Mellin-Barnes integral is given by the following formula. Let $s, \lambda$ be complex numbers, $\Re s>0,|\arg \lambda|<\pi$ and $\lambda \neq 0$. The Mellin-Barnes integral formula

$$
\Gamma(s)(1+\lambda)^{-s}=\frac{1}{2 \pi i} \int_{(c)} \Gamma(s+z) \Gamma(-z) \lambda^{z} d z
$$

is classically known (see [6, Section 14.51, p. 289, Corollary]), where $-\Re s<c<0$ and the path of integration is the vertical line $\Re z=c$.

Let $\mathbb{N}_{0}$ be the set of non-negative integers. We first obtain the following proposition.

PROPOSITION 1.2. Let $i(1 \leq i \leq r)$ be a fixed integer, and put

$$
\begin{aligned}
& A_{r, i}=\left\{\left(s_{1}, \ldots, s_{r}\right) \in \mathbb{C}^{r} \mid \Re\left(s_{r-k+1}+\cdots+s_{r}\right)>k+\mu(r-i+1 \leq k \leq r)\right\}, \\
& B_{r, i}=\left\{\left(s_{1}, \ldots, s_{r}\right) \in \mathbb{C}^{r} \mid \Re\left(s_{r-k+1}+\cdots+s_{r}\right)>k(1 \leq k \leq r-i)\right\} .
\end{aligned}
$$

Then the series (1.1) is absolutely convergent in the region $A_{r, i} \cap B_{r, i}$, uniformly in any compact subset of $A_{r, i} \cap B_{r, i}$.

This is analogous to [2, Proposition 1.1]. 


\section{THEOREM 1.3.}

(1) The series (1.1) can be continued meromorphically, as a function in $s_{1}, \ldots, s_{r}$, to the whole $\mathbb{C}^{r}$-space.

(2) The function $L_{r, i}$ is holomorphic except for the possible singularities located only on

$$
\begin{aligned}
s_{r} & =1, \\
s_{r}+s_{r-1} & =2,1,-2 n \quad\left(n \in \mathbb{N}_{0}\right), \\
s_{r}+s_{r-1}+s_{r-2} & =3-n \quad\left(n \in \mathbb{N}_{0}\right), \\
& \vdots \\
s_{r}+\cdots+s_{1} & =r-n \quad\left(n \in \mathbb{N}_{0}\right) .
\end{aligned}
$$

Our proof of Theorem 1.3 will be presented in Section 2.

Note that, when $a(m) \equiv 1(m \in \mathbb{N})$, we have $L_{r, i}\left(\left(s_{1}, \ldots, s_{r}\right) ; a\right)=\zeta_{E Z, r}\left(s_{1}, \ldots, s_{r}\right)$, and the above list of possible singularities coincides with the list given in Theorem 1.1. Masri [2] also gave the list of possible singularities in the case $i=1, r$, but when $i=r$, the singularity $s_{r}+s_{r-1}=2,1,-2 n\left(n \in \mathbb{N}_{0}\right)$ is misstated as $s_{1}+s_{2}=2,1,-2 n$ in [2].

Also, we can show that all the possible singularities listed in Theorem 1.3 are indeed the true singularities.

THEOREM 1.4. The true singularities of (1.1) lie on the subsets of $\mathbb{C}^{r}$ given by one of the following equations:

$$
\begin{aligned}
s_{r} & =1, \\
s_{r}+s_{r-1} & =2,1,-2 n \quad\left(n \in \mathbb{N}_{0}\right), \\
s_{r}+s_{r-1}+s_{r-2} & =3-n \quad\left(n \in \mathbb{N}_{0}\right), \\
& \vdots \\
s_{r}+\cdots+s_{1} & =r-n \quad\left(n \in \mathbb{N}_{0}\right) .
\end{aligned}
$$

Theorem 1.4 is a generalization of Theorem 1.1. Our proof of Theorem 1.4 is based on two methods; one is to use functional relations, which we will obtain by transforming the relevant sums (see (3.11) and (3.12)), and the other is the technique of changing variables introduced in [1]. The transformations, by which we will obtain functional relations, are different from the transformations (2.3) and (2.4) used in the proof of Theorem 1.3. These transformation arguments are the most novel part of the present paper.

Our proof of Theorem 1.4 will be presented in Section 3.

\section{Proof of Theorem 1.3}

First of all we prove the case $i=1$ by induction on $r$, and next we prove the case $i=r$ by using the result of the case $i=1$. The proof of the remaining $i=r-1, r-2, \ldots, 2$ is similar to $i=r$. Hence the proof in the case $i=r-1, r-2, \ldots, 2$ is left to the readers, and we will prove Theorem 1.3 for $i=1$ and $i=r$ in the present paper. 
When $i=1$, the series can be seen as a special case of the following multiple Dirichlet series:

$$
\sum_{m_{1}=1}^{\infty} \cdots \sum_{m_{r}=1}^{\infty} a_{1}\left(m_{1}\right) m_{1}^{-s_{1}} a_{2}\left(m_{2}\right)\left(m_{1}+m_{2}\right)^{-s_{2}} \cdots a_{r}\left(m_{r}\right)\left(m_{1}+\cdots+m_{r}\right)^{-s_{r}},
$$

which was studied by Matsumoto and Tanigawa [5], where $a_{k}\left(m_{k}\right)(1 \leq k \leq r)$ are complex coefficients. Hence the proof in the case $i=1$ is similar to that in [5].

However we should show that $L_{k, 1}(2 \leq k \leq r)$ does not have a singularity at $s_{r}+s_{r-1}=$ $1-2 n(n \in \mathbb{N})$. In fact, in the case $r=2$, by using (1.4) we have

$$
\begin{aligned}
L_{2,1}\left(\left(s_{1}, s_{2}\right) ; a\right)= & \frac{1}{s_{2}-1} L\left(s_{1}+s_{2}-1\right)+\sum_{l=0}^{N-1}\left(\begin{array}{c}
-s_{2} \\
l
\end{array}\right) L\left(s_{1}+s_{2}+l\right) \zeta(-l) \\
& +\frac{1}{2 \pi i} \int_{(N-\eta)} \frac{\Gamma\left(s_{2}+z\right) \Gamma(-z)}{\Gamma\left(s_{2}\right)} L\left(s_{1}+s_{2}+z\right) \zeta(-z) d z,
\end{aligned}
$$

where $N$ is a positive integer and $\eta$ is a small positive number. Note that we assume $\sigma_{1}>$ $1+\mu$ and $\sigma_{2}>1$. Then, the singularity of $L\left(s_{1}+s_{2}+l\right)$ is determined by $s_{1}+s_{2}=1-l$ $\left(l \in \mathbb{N}_{0}\right)$. However, $\zeta(-2 m)=0(m \in \mathbb{N})$. Hence, from $(2.1)$, we have

$$
\begin{aligned}
L_{2,1}\left(\left(s_{1}, s_{2}\right) ; a\right)= & \frac{1}{s_{2}-1} L\left(s_{1}+s_{2}-1\right)+L\left(s_{1}+s_{2}\right) \zeta(0) \\
& +\sum_{l=1}^{[N / 2]}\left(\begin{array}{c}
-s_{2} \\
2 l-1
\end{array}\right) L\left(s_{1}+s_{2}+2 l-1\right) \zeta(-2 l+1) \\
& +\frac{1}{2 \pi i} \int_{(N-\eta)} \frac{\Gamma\left(s_{2}+z\right) \Gamma(-z)}{\Gamma\left(s_{2}\right)} L\left(s_{1}+s_{2}+z\right) \zeta(-z) d z .
\end{aligned}
$$

Therefore, from (2.2), we see that $L_{2,1}$ does not have a singularity at $s_{1}+s_{2}=1-2 n$ $(n \in \mathbb{N})$. The case $r>2$ is similar to $r=2$. We have proved Theorem 1.3 for $i=1$.

Next we will prove Theorem 1.3 for $i=r$ by induction on $r$, and at first assume that $\sigma_{1}>1$ and $\sigma_{2}>1+\mu$.

When $r=2$, we have

$$
\begin{aligned}
L_{2,2}\left(\left(s_{1}, s_{2}\right) ; a\right)= & \sum_{m_{2}=1}^{\infty} a\left(m_{2}\right) m_{2}^{-s_{2}} \sum_{m_{1}=1}^{m_{2}-1} m_{1}^{-s_{1}} \\
= & \sum_{m_{2}=1}^{\infty} a\left(m_{2}\right) m_{2}^{-s_{2}} \sum_{m_{1}=1}^{\infty} m_{1}^{-s_{1}}-\sum_{m_{2}=1}^{\infty} a\left(m_{2}\right) m_{2}^{-s_{2}-s_{1}} \\
& -\sum_{m_{1}=1}^{\infty} m_{1}^{-s_{1}} \sum_{m_{2}=1}^{m_{1}-1} a\left(m_{2}\right) m_{2}^{-s_{2}} \\
= & L\left(s_{2}\right) \zeta\left(s_{1}\right)-L\left(s_{1}+s_{2}\right)-L_{2,1}\left(\left(s_{2}, s_{1}\right) ; a\right),
\end{aligned}
$$

where $\zeta$ is the Riemann zeta function. Then both the first and the second terms on the righthand side of (2.3) can be continued meromorphically to the whole $\mathbb{C}^{2}$-space. The third term on the right-hand side of (2.3) can be continued meromorphically to the whole $\mathbb{C}^{2}$-space by 
using Theorem 1.3 for $r=2$ and $i=1$. Hence our remaining task is to consider the possible singularities of $L_{2,2}$. By (2.3) we have the following possible singularities:

$$
\begin{aligned}
s_{1} & =1, \quad s_{2}=1, \\
s_{2}+s_{1} & =2,1,-2 n \quad\left(n \in \mathbb{N}_{0}\right) .
\end{aligned}
$$

When $s_{1}=1$, both the first and the third terms on the right-hand side of (2.3) are singular, but these singularities cancel each other, which can be seen by substituting (2.1) into (2.3). Hence $L_{2,2}$ is holomorphic except for the possible singularities located only on

$$
\begin{aligned}
s_{2} & =1, \\
s_{2}+s_{1} & =2,1,-2 n \quad\left(n \in \mathbb{N}_{0}\right) .
\end{aligned}
$$

Hence we have proved Theorem 1.3 for $i=r$ and $r=2$.

Next we assume the validity of Theorem 1.3 for $L_{r-1, r-1}$, and we will prove Theorem 1.3 for $L_{r, r}$. First of all, we assume that $\sigma_{j}>1(1 \leq j \leq r-1)$ and $\sigma_{r}>1+\mu$. Then we have

$$
\begin{aligned}
L_{r, r} & \left(\left(s_{1}, \ldots, s_{r}\right) ; a\right) \\
= & \sum_{m_{r}=1}^{\infty} \sum_{m_{r-1}=1}^{m_{r}-1} \cdots \sum_{m_{1}=1}^{m_{2}-1} a\left(m_{r}\right) m_{1}^{-s_{1}} m_{2}^{-s_{2}} \cdots m_{r}^{-s_{r}} \\
= & \sum_{m_{r}=1}^{\infty} \sum_{m_{r-1}=1}^{m_{r}-1} \cdots \sum_{m_{2}=1}^{m_{3}-1} \sum_{m_{1}=1}^{\infty} a\left(m_{r}\right) m_{1}^{-s_{1}} m_{2}^{-s_{2}} \cdots m_{r}^{-s_{r}} \\
& -\sum_{m_{r}=1}^{\infty} \sum_{m_{r-1}=1}^{m_{r}-1} \cdots \sum_{m_{2}=1}^{m_{3}-1} a\left(m_{r}\right) m_{2}^{-s_{1}-s_{2}} \cdots m_{r}^{-s_{r}} \\
& -\sum_{m_{r}=1}^{\infty} \sum_{m_{r-1}=1}^{m_{r}-1} \ldots \sum_{m_{2}=1}^{m_{3}-1} \sum_{m_{1}=m_{2}+1}^{\infty} a\left(m_{r}\right) m_{1}^{-s_{1}} m_{2}^{-s_{2}} \cdots m_{r}^{-s_{r}} \\
= & L_{r-1, r-1}\left(\left(s_{2}, \ldots, s_{r}\right) ; a\right) \zeta\left(s_{1}\right)-L_{r-1, r-1}\left(\left(s_{1}+s_{2}, s_{3}, \ldots, s_{r}\right) ; a\right) \\
& -\sum_{m_{r}=1}^{\infty} \sum_{m_{r-1}=1}^{m_{r}-1} \ldots \sum_{m_{2}=1}^{m_{3}-1} \sum_{m_{1}=1}^{\infty} a\left(m_{r}\right) m_{r}^{-s_{r}} \cdots m_{2}^{-s_{2}-s_{1}}\left(1+m_{1} / m_{2}\right)^{-s_{1}} .
\end{aligned}
$$

Then both the first and the second terms on the right-hand side of (2.4) can be continued meromorphically to the whole $\mathbb{C}^{r}$-space by using Theorem 1.3 for $L_{r-1, r-1}$. Put $s=s_{1}$ and $\lambda=m_{1} / m_{2}$. Then we can use the Mellin-Barnes integral for the third term on the right-hand side of (2.4). We have

$$
\begin{aligned}
& \sum_{m_{r}=1}^{\infty} \sum_{m_{r-1}=1}^{m_{r}-1} \cdots \sum_{m_{2}=1}^{m_{3}-1} \sum_{m_{1}=1}^{\infty} a\left(m_{r}\right) m_{r}^{-s_{r}} \cdots m_{2}^{-s_{2}-s_{1}}\left(1+m_{1} / m_{2}\right)^{-s_{1}} \\
& \quad=\frac{1}{2 \pi i} \int_{(c)} \frac{\Gamma\left(s_{1}+z\right) \Gamma(-z)}{\Gamma\left(s_{1}\right)} L_{r-1, r-1}\left(\left(s_{1}+s_{2}+z, \ldots, s_{r}\right) ; a\right) \zeta(-z) d z .
\end{aligned}
$$

We may assume

$$
\max \left\{-\sigma_{1},-\mu-1\right\}<c<-1 \text {. }
$$


Now we shift the path of integration to $\Re z=N-\eta$, where $N$ is a positive integer and $\eta$ is a small positive number. Counting the residues of the poles, we get

$$
\begin{aligned}
\frac{1}{2 \pi i} & \int_{(c)} \frac{\Gamma\left(s_{1}+z\right) \Gamma(-z)}{\Gamma\left(s_{1}\right)} L_{r-1, r-1}\left(\left(s_{1}+s_{2}+z, \ldots, s_{r}\right) ; a\right) \zeta(-z) d z \\
= & \frac{1}{s_{1}-1} L_{r-1, r-1}\left(\left(s_{1}+s_{2}-1, s_{3}, \ldots, s_{r}\right) ; a\right) \\
& \quad+\sum_{l=0}^{N-1}\left(\begin{array}{c}
-s_{1} \\
l
\end{array}\right) L_{r-1, r-1}\left(\left(s_{1}+s_{2}+l, s_{3}, \ldots, s_{r}\right) ; a\right) \zeta(-l) \\
& +\frac{1}{2 \pi i} \int_{(N-\eta)} \frac{\Gamma\left(s_{1}+z\right) \Gamma(-z)}{\Gamma\left(s_{1}\right)} L_{r-1, r-1}\left(\left(s_{1}+s_{2}+z, \ldots, s_{r}\right) ; a\right) \zeta(-z) d z .
\end{aligned}
$$

The last integral can be continued holomorphically to the whole $\mathbb{C}^{r}$-space since $N$ is arbitrary. Hence, by (2.4) and (2.5), we see that $L_{r, r}$ can be continued holomorphically except for the possible singularities located only on

$$
\begin{aligned}
s_{r}=1, \quad s_{1}=1, & \\
s_{r}+s_{r-1} & =2,1,-2 n \quad\left(n \in \mathbb{N}_{0}\right), \\
s_{r}+s_{r-1}+s_{r-2} & =3-n \quad\left(n \in \mathbb{N}_{0}\right), \\
\vdots & \\
s_{r}+\cdots+s_{1} & =r-1-n \quad\left(n \in \mathbb{N}_{0}\right) .
\end{aligned}
$$

However when $s_{1}=1$, the first terms on the right-hand sides of both (2.4) and (2.5) are singular, but these singularities cancel each other. Hence we have proved Theorem 1.3 for $i=r$.

\section{Proof of Theorem 1.4}

In this section, we prove that the possible singularities of (1.1) are indeed the true singularities of (1.1). First of all we consider the case $i=1$ by induction on $r$. In this case, we can prove easily by using the technique of changing variables introduced in [1]. Hence, we omit the proof. Next we prove the case $i=r$ by induction on $r$.

In the case $r=2$, from (2.3), we have

$$
L_{2,2}\left(\left(s_{1}, s_{2}\right) ; a\right)=L\left(s_{2}\right) \zeta\left(s_{1}\right)-L\left(s_{1}+s_{2}\right)-L_{2,1}\left(\left(s_{2}, s_{1}\right) ; a\right) .
$$

Then, the singularity of the first term on the right-hand side of (2.3) is determined by

$$
s_{2}=1 \text {. }
$$

Similarly, the singularities of the second term and the third term on the right-hand side of (2.3) are determined by

$$
\begin{aligned}
& s_{2}+s_{1}=1, \\
& s_{2}+s_{1}=2,1,-2 n \quad\left(n \in \mathbb{N}_{0}\right),
\end{aligned}
$$

respectively. First we claim that (3.1) is a true singularity of $L_{2,2}$. In fact, since $\zeta\left(s_{1}\right) \not \equiv 0$, (3.1) determines a true singularity of $L_{2,2}$. 
Next we claim that (3.2) and (3.3) are true singularities of $L_{2,2}$. We consider the third term on the right-hand side of (2.3). Then, from (2.1), we have

$$
\begin{aligned}
L_{2,1}\left(\left(s_{2}, s_{1}\right) ; a\right)= & \frac{1}{s_{1}-1} L\left(s_{1}+s_{2}-1\right) \\
& +\sum_{l=0}^{N-1}\left(\begin{array}{c}
-s_{1} \\
l
\end{array}\right) L\left(s_{1}+s_{2}+l\right) \zeta(-l) \\
& +\frac{1}{2 \pi i} \int_{(N-\eta)} \frac{\Gamma\left(s_{1}+z\right) \Gamma(-z)}{\Gamma\left(s_{1}\right)} L\left(s_{1}+s_{2}+z\right) \zeta(-z) d z
\end{aligned}
$$

where $N$ is a positive integer and $\eta$ is a small positive number.

The singularities of (3.3) come from the first term and the second term on the righthand side of (3.4). Actually, $s_{1}+s_{2}=2$ comes from the first term on the right-hand side of (3.4) and $s_{2}+s_{1}=1,-2 n$ come from the second term on the right-hand side of (3.4). Since $s_{2}+s_{1}=2,-2 n$ are not cancelled with other factors, $s_{2}+s_{1}=2,-2 n$ determine true singularities of $L_{2,2}$.

In the case $s_{2}+s_{1}=1$, we need to consider the second terms on the right-hand sides of (2.3) and (3.4). We note that we consider only the case $l=0$ for (3.4). Then, the second terms on the right-hand sides of (2.3) and (3.4) are not cancelled by the factor $\zeta(0)$. Hence $s_{2}+s_{1}=1$ determines a true singularity of $L_{2,2}$. Therefore we have proved Theorem 1.4 for $i=r$ and $r=2$.

Next we assume the validity of Theorem 1.4 for $L_{r-1, r-1}$, and we will prove Theorem 1.4 for $L_{r, r}$. From (2.4) and (2.5), we have

$$
\begin{aligned}
& L_{r, r}\left(\left(s_{1}, \ldots, s_{r}\right) ; a\right) \\
& =L_{r-1, r-1}\left(\left(s_{2}, \ldots, s_{r}\right) ; a\right) \zeta\left(s_{1}\right)-L_{r-1, r-1}\left(\left(s_{1}+s_{2}, s_{3}, \ldots, s_{r}\right) ; a\right) \\
& \quad-\frac{1}{s_{1}-1} L_{r-1, r-1}\left(\left(s_{1}+s_{2}-1, s_{3}, \ldots, s_{r}\right) ; a\right) \\
& \quad-\sum_{l=0}^{N-1}\left(\begin{array}{c}
-s_{1} \\
l
\end{array}\right) L_{r-1, r-1}\left(\left(s_{1}+s_{2}+l, s_{3}, \ldots, s_{r}\right) ; a\right) \zeta(-l) \\
& \quad-\frac{1}{2 \pi i} \int_{(N-\eta)} \frac{\Gamma\left(s_{1}+z\right) \Gamma(-z)}{\Gamma\left(s_{1}\right)} L_{r-1, r-1}\left(\left(s_{1}+s_{2}+z, s_{3}, \ldots, s_{r}\right) ; a\right) \zeta(-z) d z,
\end{aligned}
$$

where $N$ is a positive integer and $\eta$ is a small positive number. Then, singularities of the first, second, third and fourth terms on the right-hand side of (3.5) are determined by

$$
\left\{\begin{array}{l}
s_{r}=1, \\
s_{r}+s_{r-1}=2,1,-2 n \quad\left(n \in \mathbb{N}_{0}\right), \\
s_{r}+s_{r-1}+s_{r-2}=3-n \quad\left(n \in \mathbb{N}_{0}\right), \\
\vdots \\
s_{r}+\cdots+s_{2}=r-1-n \quad\left(n \in \mathbb{N}_{0}\right),
\end{array}\right.
$$




$$
\begin{aligned}
& \left\{\begin{array}{l}
s_{r}=1, \\
s_{r}+s_{r-1}=2,1,-2 n \quad\left(n \in \mathbb{N}_{0}\right), \\
s_{r}+s_{r-1}+s_{r-2}=3-n \quad\left(n \in \mathbb{N}_{0}\right), \\
\vdots \\
s_{r}+\cdots+s_{3}=r-2-n \quad\left(n \in \mathbb{N}_{0}\right), \\
s_{r}+\cdots+s_{2}+s_{1}=r-1-n \quad\left(n \in \mathbb{N}_{0}\right),
\end{array}\right. \\
& \left\{\begin{array}{l}
s_{r}=1, \\
s_{r}+s_{r-1}=2,1,-2 n \quad\left(n \in \mathbb{N}_{0}\right), \\
s_{r}+s_{r-1}+s_{r-2}=3-n \quad\left(n \in \mathbb{N}_{0}\right), \\
\vdots \\
s_{r}+\cdots+s_{3}=r-2-n \quad\left(n \in \mathbb{N}_{0}\right), \\
s_{r}+\cdots+s_{2}+s_{1}-1=r-1-n \quad\left(n \in \mathbb{N}_{0}\right),
\end{array}\right.
\end{aligned}
$$

and

$$
\left\{\begin{array}{l}
s_{r}=1 \\
s_{r}+s_{r-1}=2,1,-2 n \quad\left(n \in \mathbb{N}_{0}\right) \\
s_{r}+s_{r-1}+s_{r-2}=3-n \quad\left(n \in \mathbb{N}_{0}\right) \\
\vdots \\
s_{r}+\cdots+s_{3}=r-2-n \quad\left(n \in \mathbb{N}_{0}\right) \\
s_{r}+\cdots+s_{2}+s_{1}+l=r-1-n \quad\left(n, l \in \mathbb{N}_{0}\right)
\end{array}\right.
$$

respectively. First, since $\zeta\left(s_{1}\right) \not \equiv 0, s_{r}+\cdots+s_{2}=r-1-n$ of (3.6) is a true singularity of $L_{r, r}$. Also, we can prove that $s_{r}+\cdots+s_{2}+s_{1}=r-1-n$ of (3.7), (3.8) and (3.9) is a true singularity of $L_{r, r}$ by using the technique of changing variables.

Lastly, we consider the following subsets of $\mathbb{C}^{r}$ :

$$
\left\{\begin{array}{l}
s_{r}=1 \\
s_{r}+s_{r-1}=2,1,-2 n \quad\left(n \in \mathbb{N}_{0}\right), \\
s_{r}+s_{r-1}+s_{r-2}=3-n \quad\left(n \in \mathbb{N}_{0}\right) \\
\vdots \\
s_{r}+\cdots+s_{3}=r-2-n \quad\left(n \in \mathbb{N}_{0}\right) .
\end{array}\right.
$$

We will prove that (3.10) are true singularities of $L_{r, r}$ by using certain functional relations. First we claim that $s_{r}=1$ is a true singularity of $L_{r, r}$. The functional relation we use here is 
the following:

$$
\begin{aligned}
L_{r, r} & \left(\left(s_{1}, \ldots, s_{r}\right) ; a\right) \\
= & \sum_{m_{1}=1}^{\infty} \sum_{m_{2}=m_{1}+1}^{\infty} \ldots \sum_{m_{r-1}=m_{r-2}+1}^{\infty} \sum_{m_{r}=1}^{\infty} a\left(m_{r}\right) m_{1}^{-s_{1}} m_{2}^{-s_{2}} \ldots m_{r}^{-s_{r}} \\
& \quad-\sum_{m_{1}=1}^{\infty} \sum_{m_{2}=m_{1}+1}^{\infty} \ldots \sum_{m_{r-1}=m_{r-2}+1}^{\infty} \sum_{m_{r}=1}^{m_{r-1}} a\left(m_{r}\right) m_{1}^{-s_{1}} m_{2}^{-s_{2}} \ldots m_{r}^{-s_{r}} \\
= & \zeta_{E Z, r-1}\left(s_{1}, \ldots, s_{r-1}\right) L\left(s_{r}\right)-\sum_{i=1}^{r-1} L_{r-1, i}\left(\left(s_{1}, \ldots, s_{i}+s_{r}, \ldots, s_{r-1}\right) ; a\right) \\
& -\sum_{k=2}^{r-1} L_{r, k}\left(\left(s_{1}, \ldots, s_{k-1}, s_{r}, s_{k} \ldots, s_{r-1}\right) ; a\right)-L_{r, 1}\left(\left(s_{r}, s_{1}, \ldots, s_{r-1} ; a\right),\right.
\end{aligned}
$$

that is,

$$
\begin{aligned}
\zeta_{E Z, r-1}\left(s_{1}, \ldots, s_{r-1}\right) L\left(s_{r}\right) \\
=L_{r, r}\left(\left(s_{1}, \ldots, s_{r}\right) ; a\right)+\sum_{i=1}^{r-1} L_{r-1, i}\left(\left(s_{1}, \ldots, s_{i}+s_{r}, \ldots, s_{r-1}\right) ; a\right) \\
\quad+\sum_{k=2}^{r-1} L_{r, k}\left(\left(s_{1}, \ldots, s_{k-1}, s_{r}, s_{k} \ldots, s_{r-1}\right) ; a\right) \\
\quad-L_{r, 1}\left(\left(s_{r}, s_{1}, \ldots, s_{r-1} ; a\right) .\right.
\end{aligned}
$$

By the assumption for $L(s)$ and Theorem 1.1, the true singularities of the left-hand side of (3.11) lie on the subsets of $\mathbb{C}^{r}$ given by one of the following equations:

$$
\begin{aligned}
s_{r} & =1 \\
s_{r-1} & =1 \\
s_{r-1}+s_{r-2} & =2,1,-2 n \quad\left(n \in \mathbb{N}_{0}\right), \\
& \vdots \\
s_{r-1}+\cdots+s_{1} & =r-1-n \quad\left(n \in \mathbb{N}_{0}\right) .
\end{aligned}
$$

However, on the right-hand side of (3.11), only the first term has a possible singularity at $s_{r}=1$. Hence $s_{r}=1$ determines a true singularity of $L_{r, r}$.

Next we claim that $s_{r}+s_{r-1}=2,1,-2 n$ are true singularities of $L_{r, r}$. This time the functional relation we use is the following:

$$
\begin{aligned}
L_{r, r} & \left(\left(s_{1}, \ldots, s_{r}\right) ; a\right) \\
= & \sum_{m_{r}=1}^{\infty} \sum_{m_{r-1}=1}^{m_{r}-1} \sum_{m_{r-2}=1}^{\infty} \ldots \sum_{m_{2}=1}^{m_{3}-1} \sum_{m_{1}=1}^{m_{2}-1} a\left(m_{r}\right) m_{1}^{-s_{1}} m_{2}^{-s_{2}} \cdots m_{r}^{-s_{r}} \\
& -\sum_{m_{r}=1}^{\infty} \sum_{m_{r-1}=1}^{m_{r}-1} \sum_{m_{r-2}=m_{r-1}}^{\infty} \cdots \sum_{m_{2}=1}^{m_{3}-1} \sum_{m_{1}=1}^{m_{2}-1} a\left(m_{r}\right) m_{1}^{-s_{1}} m_{2}^{-s_{2}} \cdots m_{r}^{-s_{r}}
\end{aligned}
$$




$$
\begin{aligned}
= & \zeta_{E Z, r-2}\left(s_{1}, \ldots, s_{r-2}\right) L_{2,2}\left(\left(s_{r-1}, s_{r}\right) ; a\right)-L_{r-1, r-1}\left(\left(s_{1}, \ldots, s_{r-2}+s_{r-1}, s_{r}\right)\right) \\
& -\sum_{m_{r}=1}^{\infty} \sum_{m_{r-1}=1}^{m_{r}-1} \sum_{m_{r-2}=m_{r-1}+1}^{\infty} \ldots \sum_{m_{2}=1}^{m_{3}-1} \sum_{m_{1}=1}^{m_{2}-1} a\left(m_{r}\right) m_{1}^{-s_{1}} m_{2}^{-s_{2}} \cdots m_{r}^{-s_{r}} .
\end{aligned}
$$

Then, from Theorem 1.3, we see that $s_{r}+s_{r-1}=2,1,-2 n$ are possible singularities on the left-hand side of (3.12). On the right-hand side of (3.12), $s_{r}+s_{r-1}=2,1,-2 n$ only come from the first term. Also, by assumption for $L_{2,2}, s_{r}+s_{r-1}=2,1,-2 n$ are true singularities on the right-hand side of (3.12). Hence $s_{r}+s_{r-1}=2,1,-2 n$ determine true singularities of $L_{r, r}$. Similarly, for the rest of (3.10), we see that (3.10) are true singularities of $L_{r, r}$. Hence we have proved Theorem 1.3 for $i=r$.

The proof of the remaining cases $i=r-1, r-2, \ldots, 2$ is similar to $i=r$, which is left to the readers.

Acknowledgements. The author sincerely thanks Professor Kohji Matsumoto for discussions and encouragement. This paper was improved by his valuable comments. The author is supported by JSPS Research Fellowship for Young Scientists (JSPS Research Fellow DC).

\title{
REFERENCES
}

[1] S. Akiyama, S. Egami and Y. Tanigawa. Analytic continuation of multiple zeta functions and their values at non-positive integers. Acta Arith. 98 (2001), 107-116.

[2] R. Masri. Multiple Dedekind zeta functions and evaluations of extend multiple zeta values. J. Number Theory 115 (2005), 295-309.

[3] K. Matsumoto. Asymptotic expansions of double zeta-functions of Barnes, of Shintani, and Eisenstein series. Nagoya Math. J. 172 (2003), 59-102.

[4] K. Matsumoto. The analytic continuation and the asymptotic behaviour of certain multiple zeta-functions, I. J. Number Theory 101 (2003), 223-243.

[5] K. Matsumoto and Y. Tanigawa. The analytic continuation and the order estimate of multiple Dirichlet series. J. Théor. Nombres Bordeaux 15 (2003), 267-274.

[6] E. T. Whittaker and G. N. Watson. A Course of Modern Analysis. Cambridge University Press, Cambridge, 1927.

[7] Z. Wojtkowiak. Mixed Hodge structure and iterated integrals, I. Motives, Polylogarithms, and Hodge theory, Part I (Irvine, CA, 1998) (International Press Lecture Series, Vol. 3, part I). International Press, Somerville, MA, 2002, pp. 121-208.

[8] D. Zagier. Values of zeta functions and their applications. First European Congress of Mathematics, Vol. II (Progress in Mathematics, 120). Eds. A. Joseph et al. Birkhäuser, Boston, 1994, pp. 497-512.

[9] J. Zhao. Analytic continuation of multiple zeta functions. Proc. Amer. Math. Soc. 128 (2000), 1275-1283.

[10] J. Zhao. Variations of mixed Hodge structure of multiple polylogarithm. Canada. J. Math. 56 (2004), 1308-1338.

\author{
Takuya Okamoto \\ Graduate School of Mathematics \\ Nagoya University \\ Chikusa-ku \\ Nagoya 464-8602 \\ Japan
}

(E-mail:m07011y@math.nagoya-u.ac.jp) 